\title{
Comparison between Characteristics of Maxillary Right and Left Central Incisors: A Cone-beam Computed Tomography Study
}

Amal I Linjawi

\begin{abstract}
Aim: To compare the angulation of maxillary left (UL) and right (UR) incisors and the width of alveolar bone.

Materials and methods: This study was conducted using archived cone-beam computed tomography (CBCT) images of 50 male and 50 female patients. The UL and UR incisors were compared in terms of incisor/palatal plane angle, collum angle, labiopalatal crown-root position, and alveolar bone width (ABW). The comparison, with reference to gender and age, was analyzed using one-way analysis of variance and independent sample $t$ test.

Results: There is no substantial variance in the average differences of the assessed variables for UR and UL central incisors $(p>0.05)$. No significant association was found between crown labial to root and root labial to crown positions for both central incisors $(p>0.05)$. Statistical analyses revealed that tooth type has no significant association with the central incisors-related variables. Alveolar bone width, at various areas assessed, showed significant relation to gender. On the other hand, incisor/palatal plane angle and ABW at the cementoenamel junction and at level of Point A (subnasale) were significantly affected by age.

Conclusion: Gender can significantly affect the development of ABW. Also, incisor/palatal plane angle and ABW at certain areas are correlated with age.

Clinical significance: Understanding the similarities or differences between right and left maxillary central incisors may give better indication if cephalometric images are accurate in attaining such measurements. This in turn will also help orthodontist to choose the proper tool for treatment decision-making related to incisor tooth movement.

Keywords: Alveolar bone width, Collum angle, Cone-beam computed tomography, Crown-to-root angle, Incisors' characteristics.

The Journal of Contemporary Dental Practice (2020): 10.5005/jp-journals-10024-2874
\end{abstract}

\section{INTRODUCTION}

Orthodontic treatment can be attained by teeth movement in its desired place. As such, suitable support for alveolar bone is highly needed for efficient movement of teeth and steady position. ${ }^{1}$ In order to achieve this, premolar extraction and anterior retraction are required for many malocclusion problems. Treating such cases requires controlling the torque of the incisor while retracting them. ${ }^{2}$ Understanding and examining the periodontium and tooth structure of the incisors before retraction are necessary to envisage limitations and possibilities of fenestrations, loss of alveolar bone, or dehiscence during incisor retraction. ${ }^{1-3}$

Accurate diagnostic imaging is an indispensable necessity in order to obtain the accurate diagnosis and ideal action as well as in monitoring the development and result of treatment. ${ }^{4,5}$ Cephalometric radiographs are two-dimensional projection of the facial skeleton in the mid-sagittal plane. However, it does not provide a factual scale for the incisor inclinations and alveolar bone width (ABW). ${ }^{3,6}$ As such, old-style radiographic images, like periapical radiographs, panoramic views, and cephalograms, are deemed less precise in assessing bone structure., ${ }^{3,7}$ A study conducted by Fuhrmann revealed a common overestimation of the width of labiolingual bone on the lateral cephalogram in comparison to physical dimensions of the concrete samples. ${ }^{2,3,8}$ Likewise, cephalometric examination has other restrictions such as $2 \mathrm{D}$ representation of anatomical structures as well as image distortion due to errors related to $\mathrm{X}$-ray equipment or variations in
Orthodontic Department, Faculty of Dentistry, King Abdulaziz University, Jeddah, Kingdom of Saudi Arabia

Corresponding Author: Amal I Linjawi, Orthodontic Department, Faculty of Dentistry, King Abdulaziz University, Jeddah, Kingdom of Saudi Arabia, Phone: +966 504155573, e-mail: ailinjawi@kau.edu.sa

How to cite this article: Linjawi Al. Comparison between Characteristics of Maxillary Right and Left Central Incisors: A Cone-beam Computed Tomography Study. J Contemp Dent Pract 2020;21 (7):723-727.

Source of support: Nil

Conflict of interest: None

patient's head positioning. ${ }^{6}$ In the anterior region, the true scale and inclination of the right and left incisors might be mislead by such overlap of anatomical structures.

In contrast, three-dimensional cone-beam computed tomography (CBCT) was described to be more accurate in assessing incisors' inclinations and morphology of alveolar bone. ${ }^{6,9} \mathrm{CBCT}$ is deemed advantageous in the assessment of osseous condition around teeth due to certain factors such as low radiation dose, low cost, convenience, and accuracy. ${ }^{10,11}$ Cone-beam computed tomography could be utilized as a support in case analysis and treatment preparation, as it helps in minimizing difficulties in dental procedures. ${ }^{12-14}$ Cone-beam computed tomography is extensively utilized in the assessment of width and size of the bones in maxillofacial and oral areas. ${ }^{13,15}$ 
Disparities in incisors' angulations, alveolar bone thickness, and incisive canal morphology are challenging variables that can affect the movement of maxillary incisors. ${ }^{1,13,16-18}$ Cone-beam computed tomography studies revealed a thicker alveolar bone on the palatal side when compared to the labial side and at the apical level than the coronal level of the central incisors. ${ }^{1,13,18}$ Lee et al. ${ }^{13}$ found that the majority of participants had $<2 \mathrm{~mm}$ of $A B W$ at the labial side at 3 and $5 \mathrm{~mm}$ apical to central incisors' cementoenamel junction (CEJ). No substantial variation in the finding among male and female in terms of ABW was reported. Several studies reported positive association between inclination of central incisor and morphology of alveolar bone. ${ }^{17}$

Given the availability of limited information on the relationship between the right and the left maxillary incisors' angulation and ABW, this study assessed the correlation between the angulation of both incisors and the width of the surrounding alveolar bone. The incisors' angulations assessed were: the incisor to palatal plane angle, the crown to root angle, which is called the collum angle, and the crown to root position. Such angulation in addition to measuring the surrounding alveolar bone are important measures to know when deciding tipping, torqueing, and bodily movement of incisors. In addition, understanding the similarities or differences between right and left maxillary central incisors may give better indication if cephalometric images are accurate in attaining such measurements. This in turn will also help orthodontist to choose the proper tool needed for treatment decisions related to incisor tooth movement.

\section{Materials and Methods}

\section{Study Design}

This study, which utilized a cross-sectional approach, was conducted using CBCT archived records of adult patients seeking treatment at the Faculty of Dentistry of King Abdulaziz University, Jeddah, Kingdom of Saudi Arabia. Ethical approval was obtained from the ethical committee of the same institution [ethical no. 100-06-19].

\section{Sample Characteristics}

The criteria for inclusion in this study were: (1) CBCT images for at least the maxilla was taken; (2) no orthodontic treatment history; (3) no dental anomalies related to the maxillary incisors including missing or supernumerary teeth; (4) no dental treatment to maxillary incisors; (5) no maxillary incisor trauma history; and (7) no congenital abnormalities including cleft lip and palate.

The sample was grouped according to gender and age categories. Four age-groups were identified: less than or equal to $20,20-40,40-60$, and greater than 60 years of age.

\section{Cone-beam Computed Tomography Images}

In terms of CBCT images, the subsequent settings were used: normal mode with specifications of 6.8 seconds, $4.10 \mathrm{mGy}, 90 \mathrm{kV}$, and $4 \mathrm{~mA}$; slice thickness of $0.147 \mathrm{~mm}$; field of view of $81 \times 74 \mathrm{~mm}$; and voxel size of $0.146 \mathrm{~mm}$. Image acquisition was done with the Frankfort horizontal plane parallel to the floor. Images were then saved as DICOM file, and the sagittal view was extracted as well as evaluated using OnDemand 3D Imaging software (Seoul, Korea).

\section{Measurements}

Linear and angular measurements of the maxillary left (UL) and right (UR) central incisors were assessed in the sagittal plane for the following variables (Fig. 1):

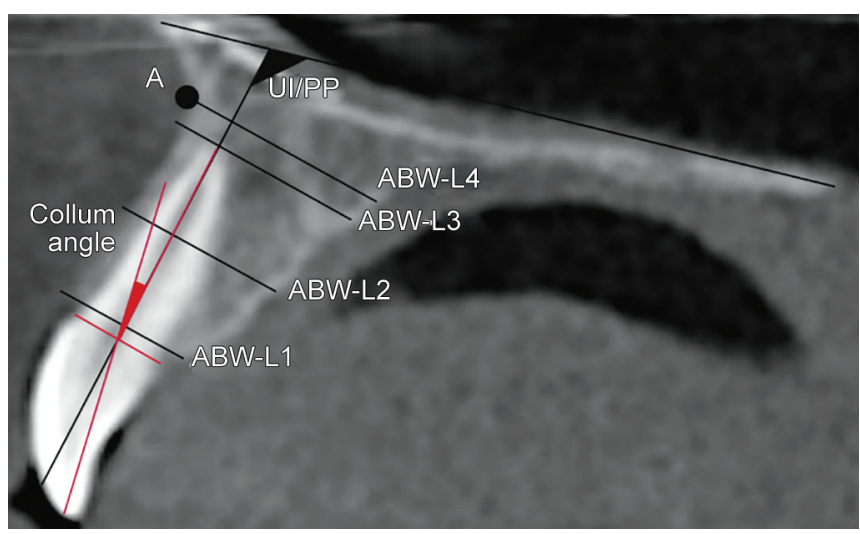

Fig. 1: UI/PP [black lines]: incisor to palatal plane angle. Collum angle [red lines]: crown-root angle. Alveolar bone width (ABW) [black lines]: lines drawn from palatal to labial surface of central incisor perpendicular to its long axis; ABW-L1: at cementoenamel junction (CEJ), ABW-L2: at mid-root level at half the distance between $L 1$ and L3, ABW-L3: at level of the root apex and ABW-L4: at the level of Point A (subnasale)

- Incisor/palatal plane angle: the angle between the long axis of the central incisor and the palatal plane.

- Collum angle: crown-root angle is the angle created by the following two lines:

- A line drawn from the crown tip passing through a mid-point at the cervical line of the tooth.

- A line drawn from the root tip passing through a mid-point at the cervical line of the tooth.

- Crown-root position: to assess the crown to root position and categorized as follows.

$1=$ crown tip is labial to the root tip.

$2=$ crown tip is palatal to the root tip.

- Alveolar bone width: The ABW at the right and left central incisors was determined by drawing a line from the palatal to the labial perspective perpendicular to the long axis of the tooth. The ABW was measured at four levels: L1 —at CEJ, L2 - at mid-root level by measuring half the distance between $\mathrm{L} 1$ and L3, L3 - at level of the root apex, and L4 - at the level of Point A (subnasale).

\section{Measurement Error}

A single examiner, the author who is an orthodontist, performed all measurements, which were repeated after a 2-week break. No substantial association between the 2 sets $(p<0.05)$ as well as within-class correlation constant of 0.81 , which reveals a good consistency of data, was determined after performing an independent $t$ test that linked the first and second sets of the measurements.

\section{Statistical Analysis}

Calculations of mean as well as standard deviations were performed for all sets of data. Assessment concerning UR and UL incisors for all variables assessed was performed according to gender using paired $t$ test. Comparison was also performed according to age categories using post hoc Tukey's test and one-way analysis of variance. Correlations among parameters were studied using Bonferroni correction for multiple comparisons and Pearson correlation analysis. Statistical analyses were determined via SPSS 22.0 computer software package (IBM, Armonk, NY, USA). The confidence level for analyses was set at $p<0.05$. 
Table 1: Mean and standard deviations of the assessed variable for the UR and UL central incisors $(n=100)$

\begin{tabular}{lllll}
\hline & $\begin{array}{l}\text { UR central } \\
\text { incisor, mean } \\
(S D)\end{array}$ & $\begin{array}{l}\text { UL central } \\
\text { incisor, mean } \\
\text { (SD) }\end{array}$ & $\begin{array}{l}\text { Mean difference } \\
\text { (right-left), } \\
\text { Mean (SD) }\end{array}$ & p value \\
\hline $\begin{array}{l}\text { Incisor/PP } \\
\text { Collum }\end{array}$ & $115.69(8.07)$ & $116.69(9.46)$ & $1.00(6.76)$ & 0.142 \\
angle & $3.43(2.50)$ & $3.47(2.98)$ & $-0.04(3.21)$ & 0.894 \\
ABW L1 & $7.58(0.77)$ & $7.55(0.81)$ & $0.03(0.66)$ & 0.636 \\
ABW L2 & $8.24(1.08)$ & $8.15(1.20)$ & $0.08(0.80)$ & 0.301 \\
ABW L3 & $9.03(1.90)$ & $8.90(1.81)$ & $0.13(1.39)$ & 0.366 \\
ABW L4 & $10.49(2.18)$ & $10.43(2.37)$ & $0.06(1.51)$ & 0.694 \\
\hline
\end{tabular}

UR, upper right central incisor; UL, upper left central incisor; PP, palatal plane; $A B W$, alveolar bone width; L1, at CEJ; L2, mid-root; L3, at root apex. Significance level at $p<0.05$

Table 3: $p$ values and significant differences in the assessed variable between the UR and UL central incisors according to gender, age, and tooth type

\begin{tabular}{llll}
\hline & $\begin{array}{l}\text { According to } \\
\text { gender }\end{array}$ & $\begin{array}{l}\text { According to } \\
\text { age }\end{array}$ & $\begin{array}{l}\text { According to } \\
\text { tooth type }\end{array}$ \\
\hline Incisor/PP & 0.432 & $0.028^{*}$ & 0.422 \\
Collum angle & 0.142 & 0.576 & 0.911 \\
Crown-root position & 0.151 & 0.818 & 0.841 \\
ABW L1 & $0.005^{* *}$ & 0.080 & 0.779 \\
ABW L2 & $0.000^{* * *}$ & 0.474 & 0.608 \\
ABW L3 & $0.000^{* * *}$ & 0.200 & 0.631 \\
ABW L4 & $0.000^{* * *}$ & $0.032^{*}$ & 0.853 \\
\hline
\end{tabular}

UR: upper right central incisor, UL: upper left central incisor

PP, palatal plane; ABW, alveolar bone width; L1, at CEJ; L2, mid-root; L3, at root apex. Significance level: ${ }^{*} p<0.05,{ }^{* *} p<0.01,{ }^{* * *} p<0.001$

\section{Results}

Archived CBCT images of 100 patients, 50 males and 50 females, were utilized in this study. Assessment of UR and UL central incisors were conducted, and mean difference was determined. Low mean differences were computed for ABW at various levels. On the other hand, the mean difference in the collum angle provided a value of -0.04 . A mean difference of 1.00 was computed for incisor/palatal plane angle. At $95 \%$ confidence level, no substantial association was determined in the mean variances of the assessed variables for UR and UL central incisors, as shown in Table 1.

Crown-root position for UR and UL incisors were also assessed, with reference to age and gender. For maxillary right (UR) central incisor, crown labial to root position were present at 29\%, whereas root labial to crown were common in $71 \%$ of the participants. On the other hand, $70 \%$ of the sample had root labial to crown position, while crown labial to root position was found in the remaining $30 \%$ for UL central incisor. There was no significant difference in crown-root positions between UR and UL central incisors at level of significance of $p<0.05$, as provided in Table 2 .

Age, gender, and tooth type were also considered in the assessment of UR and UL central incisors. Statistical analyses revealed that tooth type has no significant association with the variables related to UR and UL central incisors. Alveolar bone width at various areas assessed was found to be significantly related to gender. On the other hand, incisor/palatal plane angle, and ABW at
Table 2: Number and percentages of crown-root position for UR and UL central incisors with reference to gender and age $(n=100)$

\begin{tabular}{llll}
\hline & $\begin{array}{l}\text { UR central } \\
\text { incisor }\end{array}$ & UL central incisor & $p$ \\
\hline Crown labial to root & $29(29 \%)$ & $30(30 \%)$ & 0.819 \\
Root labial to crown & $71(71 \%)$ & $70(70 \%)$ & \\
\hline
\end{tabular}

UR, upper right central incisor; UL, upper left central incisor Significance level at $p<0.05$

the CEJ (L1) and at level of Point A (subnasale) [L4] were significantly affected by age, as shown in Table 3 .

\section{Discussion}

In a related study on Chinese adults, Zhou et al. ${ }^{11}$ found that the maxillary anterior labial bone was paper-thin, particularly at $3 \mathrm{~mm}$ beneath the CEJ and mid-root areas. Likewise, a more curved labial bone contour was present at the central incisor. In addition, the curvature angle was found to be minimal in participants with retruded maxillae as effected by the lump on the labial bone at the central incisor. As such, they suggested that angulating the implant apex to the palatal side at the upper central incisor is deemed necessary.

The relationship between thickness of alveolar and buccal bones at the central incisors were determined by Uner et al. ${ }^{19}$ using CBCT. Their study revealed that the absence of substantial association amid the alveolar bone width of the left and right central incisors. However, male participants were found to have thicker alveolar bone when compared to female counterparts. Hence, it can be suggested that gender portrays a noteworthy part in the characterization of ABW, as shown in Table 3.

However, different results on the relationship between bone thickness, age, and gender was revealed by the study of Nowzari et al. ${ }^{20}$ Results showed that the preliminary maxillary bone width has substantial influence on the retorting level of soft tissue and facial bone after tooth removal and direct implant assignment. In this study, bone thickness was found to have no association with age and gender.

A related study was also conducted by Panda et al. ${ }^{16}$ which evaluated the anterior maxillary bone width as well as the incisive canal using $\mathrm{CBCT}$ for direct implant assignment. Results showed that gender and age were two major characteristics that affected the incisive canal as well as the amount of bone anterior to it. This study reported that males have thicker anterior maxilla when compared to female counterparts. This indicates that more precautionary procedures are deemed essential for females during surgical procedures. Anterior maxillary bone thickness also declines with progressing age as to which could be associated with anterior teeth loss upon aging. ${ }^{16,21-23}$

Likewise, linear regression analysis performed by $\mathrm{Yu}$ et al. specified that the adjacent profile of alveolar bone is interrelated to the lower central incisor. ${ }^{17}$ This suggests that tooth inclination is marked by the morphology of the alveolar bone. In addition, increment of the lower arch length is a consequence of the proclination of lower incisors. This could generate dilemma with organization among lower and upper arches. Adequate withdrawal of the upper incisors could, in turn, be restricted in closing gaps in extraction. ${ }^{17,24}$

The dimension and morphology of the alveolar ridge of anterior maxilla were also measured by Zhang et al. ${ }^{18}$ using CBCT for treatment preparation. Fifty-one participants with complete 
set of teeth at right maxilla were screened for this study. They found out the increment in alveolar width from coronal to apical path for each tooth as well as the absence of substantial correlation in ridge stature between teeth. Likewise, male participants had significantly thicker ridge in comparison to female counterparts in terms of central incisors, lateral incisors, and canine teeth. This report was also consistent with the findings of Schropp et al. ${ }^{25}$

The current finding showed no significant difference between the right and the left maxillary incisors in the measurement assessed. Based on that, it can be said that the cephalometric measurements on the maxillary incisors' angulations is accurate enough to make treatment decisions. Results also showed that the alveolar bone width is thinner in females than in males and is affected by age at the level of the apex. This highlights the importance of assessing the ABWs surrounding the teeth when deciding their movement. Such measurements are more clearly defined on CBCTs than cephalometric radiographs. Thus, it is recommended to use $C B C T$ for better assessment and diagnosis of orthodontic cases.

\section{Conclusion}

Assessment of UR and UL central incisors were conducted, and the mean difference revealed low mean differences for alveolar bone width at various levels. Crown-root position for UR and UL incisors were also assessed, with reference to age and gender. Results revealed no substantial association between crown labial to root and root labial to crown positions for both UR and UL central incisors. Likewise, alveolar bone width at various areas assessed was found to be significantly related to gender. Related studies reported that the bone thickness of anterior maxilla in males is greater than that of females indicating the need for more precautionary procedures for females during surgical procedures. Anterior maxillary bone thickness also declines with progressing age as to which could be associated to anterior teeth loss upon aging. Based on the results, incisor/palatal plane angle and ABW at the CEJ (L1) and at the level of Point A (subnasale) [L4] were significantly affected by age.

\section{Declarations}

\section{Ethics Approval}

Ethical approval was done by the Ethics Committee of King Abdulaziz University, Faculty of Dentistry, Jeddah, Saudi Arabia [ethical no. 100-06-19].

\section{Author's Contributions}

All designed the study, collected patients' CBCT data, analyzed and interpreted the results, and wrote the manuscript.

\section{References}

1. Kook YA, Kim G, Kim Y. Comparison of alveolar bone loss around incisors in normal occlusion samples and surgical skeletal class III patients. Angle Orthod 2012;82(4):645-652. DOI: 10.2319/070111424.1.

2. Nahm KY, Kang JH, Moon SC, et al. Alveolar bone loss around incisors in Class I bidentoalveolar protrusion patients: A retrospective three-dimensional cone beam CT study. Dentomaxillofac Radiol 2012;41(6):481-488. DOI: $10.1259 / \mathrm{dmfr} / 30845402$.

3. Tian YL, Liu F, Sun HJ, et al. Alveolar bone thickness around maxillary central incisors of different inclination assessed with cone-beam computed tomography. Korean J Orthod 2015;45(5):245-252. DOI: 10.4041/kjod.2015.45.5.245.

4. Kapila S, Conley RS, Harrell Jr WE. The current status of cone beam computed tomography imaging in orthodontics. Dentomaxillofac Radiol 2011;40(1):24-34. DOI: 10.1259/dmfr/12615645.

5. Shortliffe E, Perreault LE, Wiederhold G, et al. Medical informatics: Computer applications in health care and biomedicine. 2nd ed., New York: Springer; 2001.

6. Zamora N, Llamas JM, Cibrián R, et al. Cephalometric measurements from $3 \mathrm{D}$ reconstructed images compared with conventional $2 \mathrm{D}$ images. Angle Orthod 2011;81(5):856-864. DOI: 10.2319/121210-717.1.

7. Lupi JE, Handelman CS, Sadowsky C. Prevalence and severity of apical root resorption and alveolar bone loss in orthodontically treated adults. Am J Orthod Dentofac Orthop 1996;109(1):28-37. DOI: 10.1016/ S0889-5406(96)70160-9.

8. Fuhrmann R. Three-dimensional interpretation of labiolingual bone width of the lower incisors. Part II. J Orofac Orthop 1996;57(3):168-185. DOI: $10.1007 / B F 02191880$.

9. Leung CC, Palomo L, Griffith R, et al. Accuracy and reliability of cone-beam computed tomography for measuring alveolar bone height and detecting bony dehiscences and fenestrations. Am J Orthod Dentofac Orthop 2010;137(4 Suppl):S109-S119. DOI: 10.1016/j. ajodo.2009.07.013.

10. Timock AM, Cook V, McDonald T, et al. Accuracy and reliability of buccal bone height and thickness measurements from cone-beam computed tomography imaging. Am J Orthod. Dentofac Orthop. 2011;140(5):734-744. DOI: 10.1016/j.ajodo.2011.06.021.

11. Zhou Z, Chen W, Shen M, et al. Cone beam computed tomographic analyses of alveolar bone anatomy at the maxillary anterior region in Chinese adults. J Biomed Res 2014;28(6):498-505.

12. Braut $\mathrm{V}$, Bornstein $M M$, Belser $\mathrm{U}$, et al. Thickness of the anterior maxillary facial bone wall-A retrospective radiographic study using cone beam computed tomography. Int J Periodontics Restorative Dent 2011;31(2):125-131.

13. Lee JE, Jung CY, Kim Y, et al. Analysis of alveolar bone morphology of the maxillary central and lateral incisors with normal occlusion. Medicina 2019;55(9):565. DOI: 10.3390/medicina55090565.

14. Lee JE, Lee YJ, Jin SH, et al. Topographic analysis of the mandibular symphysis in a normal occlusion population using cone-beam computed tomography. Exp Ther Med 2015;10(6):2150-2156. DOI: 10.3892/etm.2015.2842.

15. Fu JH, Wang HL. Reliability of volumetric imaging software for conebeam computed tomography - Influence of medical education. Dentomaxillofac Radiol 2013;22:182-186.

16. Panda M, Shankar T, Raut A, et al. Cone beam computerized tomography evaluation of incisive canal and anterior maxillary bone thickness for placement of immediate implants. J Indian Prosthodont Soc 2018;18(4):356-363. DOI: 10.4103/jips.jips_167_18.

17. Yu Q, Pan XG, Ji GP, et al. The association between lower incisal inclination and morphology of the supporting alveolar bone -- $\mathrm{A}$ cone-beam CT study. Int J Oral Sci 2009;1(4):217-223. DOI: 10.4248/ IJOS09047.

18. Zhang W, Skrypczak A, Weltman R. Anterior maxilla alveolar ridge dimension and morphology measurement by cone beam computerized tomography (CBCT) for immediate implant treatment planning. BMC Oral Health 2015;15:65. DOI: 10.1186/s12903-015-00551.

19. Uner DD, Izol BS, Gorus Z. Correlation between buccal and alveolar bone widths at the central incisors according to cone-beamcomputed tomography. Niger J Clin Pract 2019;22(1):79-84.

20. Nowzari H, Molayem S, Chiu CH, et al. Cone beam computed tomographic measurement of maxillary central incisors to determine prevalence of facial alveolar bone width $=>2 \mathrm{~mm}$. Clin Implant Dent Relat Res 2012;14(4):595-602. DOI: 10.1111/j.1708-8208.2010. 00287.x.

21. Artzi Z, Nemcovsky CE, Bitlitum I, et al. Displacement of the incisive foramen in conjunction with implant placement in the anterior maxilla without jeopardizing vitality of nasopalatine nerve 
and vessels: A novel surgical approach. Clin Oral Implants Res 2000;11(5):505-510. DOI: 10.1034/j.1600-0501.2000.011005505.x.

22. Mardinger O, Namani-Sadan N, Chaushu G, et al. Morphologic changes of the nasopalatine canal related to dental implantation: A radiologic study in different degrees of absorbed maxillae. J Periodontol 2008;79(9):1659-1662. DOI: 10.1902/jop.2008. 080043.

23. Tözüm TF, Güncü GN, Yıldırım YD, et al. Evaluation of maxillary incisive canal characteristics related to dental implant treatment with computerized tomography: A clinical multicenter study. J. Periodontol. 2012;83(3):337-343. DOI: 10.1902/jop.2011.110326.

24. Sangcharearn $\mathrm{Y}, \mathrm{Ho}$ C. Effect of Incisor Angulation on Overjet and Overbite in Class II Camouflage Treatment. A typodont study. Angle Orthod 2007;77(6):1011-1018. DOI: 10.2319/111206-460.1.

25. Schropp L, Wenzel A, Kostopoulos L, et al. Bone healing and soft tissue contour changes following single-tooth extraction: A clinical and radiographic 12-month prospective study. Int. J. Periodontics Restorative Dent 2003;23(4):313-323. 